\title{
Accelerated Cholesteryl Ester Transfer in Plasma of Patients with Hypercholesterolemia
}

\author{
John D. Bagdade, Mary C. Ritter, and P. V. Subbaiah \\ Departments of Medicine and Biochemistry, Rush Medical College, Chicago, Illinois 60612
}

\section{Abstract}

To discern the mechanism(s) that underlie abnormal cholesteryl ester transfer (CET) in patients with hypercholesterolemia, we have studied this dysfunctional step in reverse cholesterol transport in 13 subjects with genetically heterogeneous forms of hypercholesterolemia (HC). In all HC patients, the mass of CE transferred in whole plasma from HDL to VLDL and LDL increased rapidly initially and was significantly greater than in controls at 1,2 , and $4 \mathrm{~h}(P<0.005)$.

To further characterize this disturbance, we performed a series of recombination experiments. Combining $\mathrm{HC} d<1.063$ containing acceptor VLDL + LDL with the $d>1.063$ fraction from controls containing donor HDL + CE-transfer protein (CETP) and not the converse combination showed the same characteristics of accelerated CET noted with intact HC plasma, indicating that abnormal transfer was associated with the HC acceptor lipoproteins. When HC VLDL and its subfractions and LDL were isolated separately and then combined with control $d>1.063$ fractions, accelerated CET was only

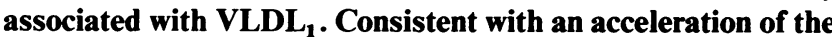
neutral lipid transfer reaction occurring between HDL and $V_{L D L}$ in $H C$ in vivo, we found that the triglyceride/CE ratio was decreased in $\mathrm{HC} \mathrm{VLDL}_{1}(P<0.001)$, and increased in HDL $(P<0.25)$. CETP mass was significantly increased in HC plasma (HC $2.3 \pm 4 \mu \mathrm{g} / \mathrm{ml}$ vs. control $1.3 \pm 0.3 \mu \mathrm{g} / \mathrm{ml}$; mean \pm SD; $P<0.025)$.

This series of observations demonstrate that CET is accelerated in the plasma of $\mathrm{HC}$ patients, and this disturbance results from dysfunction of the $\mathrm{VLDL}_{1}$ subfraction rather than an elevation of CETP levels. Since an abnormality of this type in vivo can lead to the accumulation of potentially atherogenic $\mathrm{CE}$ enriched apoB-containing lipoproteins in plasma, it may be an additional previously unrecognized factor that increases cardiovascular risk in HC patients. (J. Clin. Invest. 1991. 87:12591265.) Key words: hypercholesterolemia • cholesteryl ester transfer • cholesteryl ester transfer protein

Introduction

During the heteroexchange of neutral lipids in plasma, cholesteryl esters from HDL are exchanged for triglyceride from $\operatorname{VLDL}(1,2)$. This process is important not only because it is a

Address correspondence and reprint requests to Dr. John D. Bagdade, Section of Endocrinology and Metabolism, Rush-Presbyterian-St. Luke's Medical Center, 1653 West Congress Parkway, Chicago, IL 60612.

Received for publication 6 November 1989 and in revised form 25 October 1990.

J. Clin. Invest.

(c) The American Society for Clinical Investigation, Inc. $0021-9738 / 91 / 04 / 1259 / 07 \quad \$ 2.00$

Volume 87, April 1991, 1259-1265 key step in reverse cholesterol transport, but also because it provides a pathway for the redistribution, reutilization, as well as the eventual excretion of cholesteryl esters generated in plasma on HDL by the lecithin-cholesterol acyltransferase $(\text { LCAT })^{1}$ reaction. Disturbances in cholesterol ester transfer (CET) could have atherogenic consequences. If, for example, CET were inhibited and, as a result, the movement of cholesterol from tissues to HDL were retarded, it might predispose to the accumulation of cholesterol in arterial tissues. On the other hand, if CET were increased, and the acceptor apo B-containing lipoproteins became abnormally enriched in $\mathrm{CE}$, the physical properties and metabolism of these particles may be altered.

We recently have observed that patients with genetically heterogeneous forms of hypercholesterolemia have disturbances in lipoprotein composition which may facilitate CET (3). Specifically, we have found that their VLDL is enriched in free cholesterol and this abnormality has been reported by Morton to promote CE accumulation in VLDL (4). Moreover, our observation that the core lipid content of VLDL was enriched in CE compared with triglyceride (TG) implied that CET was increased in vivo. To determine whether CET was in fact accelerated as our compositional studies suggested, we have studied CET in a group of hypercholesterolemic patients.

\section{Methods}

Human subjects. 13 subjects ( 5 male; 8 female) with hypercholesterolemia and normal fasting triglyceride levels (Cholesterol, $304 \pm 30 \mathrm{mg}$ / dl; TG, $128 \pm 43 \mathrm{mg} / \mathrm{dl}$; HDL-cholesterol (C), $54 \pm 11 \mathrm{mg} / \mathrm{dl}$; mean $\pm \mathrm{SD}$ ) were studied (mean age, $44.1 \pm 11.6 \mathrm{yr}$; mean $\pm \mathrm{SD}$; range 21-50 yr). Al participants had fasting cholesterol levels in the untreated state measured on two or more occasions that were greater than two standard deviations of the mean defined by Lipid Research Clinics standards (5) for their age and sex. Two female patients had heterozygous familial hypercholesterolemia; 9 had familial combined hyperlipidemia based on a positive family history of hyperlipidemia and premature cardiovascular disease; and two had polygenic forms of hypercholesterolemia The apo $E$ phenotypes of 11 of 13 of these subjects were: 6 had $E_{3} / E_{3}$ and $5 \mathrm{had} \mathrm{E}_{4} / \mathrm{E}_{3}$ apoE isoforms. No effort was made to characterize the molecular basis for cholesterol elevation in any patient. Informed consent was obtained. All patients had followed American Heart Association Phase I diets for at least 4 mo before the study. None had renal disease, none were vegetarians, cigarette smokers, athletes, none took drugs known to affect lipid metabolism, or had diabetes. The normolipidemic control subjects (Cholesterol, $163 \pm 22 \mathrm{mg} / \mathrm{dl}$; TG, 107 \pm 38 $\mathrm{mg} / \mathrm{dl}$; HDL-C, $55 \pm 9 \mathrm{mg} / \mathrm{dl}$; mean $\pm \mathrm{SD}$ ) were taking no medications and were matched for sex, approximate age, weight, level of physical activity, and smoking habits. Venous blood samples were collected after an overnight fast in Na EDTA-containing tubes and plasma was separated promptly by low speed centrifugation. Enzymatic kit procedures were employed to later quantitate cholesterol (BoehringerMannheim Inc., Indianapolis IN) and triglyceride (Sigma Chemical

1. Abbreviations used in this paper: CETP, cholesteryl ester transfer protein; FC, free cholesterol; FFA, free fatty acids; HC, hypercholesterolemia; LCAT, lecithin-cholesterol acyltransferase; TG, triglyceride. 
Co., St. Louis, MO) in an aliquot of plasma which was frozen at $-20^{\circ} \mathrm{C}$, and from this same aliquot HDL-C also was measured by a standardized Lipid Research Clinic procedure (6). Free cholesterol was estimated with the same components of the cholesterol kit except that CE hydrolase was omitted. Cholesteryl ester was calculated from the difference between total and free cholesterol. For lecithin determination, 0.3-ml aliquots of VLDL, LDL, and HDL were removed, mixed with $0.1 \mathrm{ml}$ of $0.15 \mathrm{M} \mathrm{NaCl}-1 \mathrm{mM}$ EDTA solution, and extracted by the Bligh and Dyer procedure (7). All lipid extracts were spotted on activated silica gel (E. Merck, Darmstadt, FRG) thin layer plates $(0.5 \mathrm{~mm}$ thickness), and lecithin separated from the other major phospholipids using a solvent system of chloroform/methanol/acetic acid/water, 25:15:4:2 by volume. The lecithin spots were scraped into glass tubes and the lipid phosphorus determined by the modified Bartlett's procedure (8).

Cholesteryl ester transfer in incubated plasma. The mass transfer of cholesteryl ester from HDL to apo B-containing lipoproteins was measured during incubation at $37^{\circ} \mathrm{C}$ in a metabolic shaker in the presence of $1.5 \mathrm{mM}$ dithio-bis-dinitrobenzoic acid (DTNB) to inhibit plasma LCAT as previously described (9). Aliquots of plasma were removed before and at 1,2, 4, and $6 \mathrm{~h}$, chilled on ice, and VLDL + LDL were precipitated with 0.1 vol of heparin/ $/ \mathrm{MnCl}_{2}$ to give final concentrations of $\mathrm{MnCl}_{2}(0.092 \mathrm{M})$ and heparin $(1.3 \mathrm{mg} / \mathrm{ml})(10) . \mathrm{MnCl}_{2}$ at this concentration has been found to not precipitate a significant quantity of apo E-containing HDL (11). At each sampling interval, the mass of free and total cholesterol present in the supernatant were measured and the amount of CE transferred into the apo B-containing lipoproteins was calculated from the difference between the two values. The mass of CE transferred at each time interval was determined by subtracting this value from zero-time CE in HDL. Cholesteryl ester transfer protein (CETP) mass was measured in 11 of 12 subjects by radioimmunoassay in the laboratory of Dr. Yves Marcel at the Clinical Research Institute of Montreal (12).

Lipoprotein fractionation. In the series of recombination experiments performed, plasma was obtained from 6 of the 13 hypercholesterolemic patients ( 3 male, 3 female) and 6 normolipidemic control subjects of the same sex and spun at a density of 1.063 to separate the VLDL and LDL (top) and HDL + VHDL (bottom) fractions. When it was apparent that the accelerated CET observed in the intact plasma of hypercholesterolemic subjects was associated with their top fraction, VLDL $(<1.006 \mathrm{~g} / \mathrm{ml})$ and LDL $(1.006-1.063 \mathrm{~g} / \mathrm{ml})$ from the same subjects were isolated from plasma by sequential preparative ultracen- trifugation at $10^{\circ} \mathrm{C}$ in a Beckman 40.3 rotor (Beckman Instruments, Inc., Palo Alto, CA) at 40,000 rpm, and then combined with the $d$ $>1.063$ fractions of controls at concentrations equivalent to plasma in similar experiments to assess their respective contributions. During this isolation procedure, no LCAT inhibitors were employed. In $12 \mathrm{HC}$ patients, VLDL subfractions $\left(\mathrm{VLDL}_{1}, \mathrm{VLDL}_{2}\right.$, and $\mathrm{VLDL}_{3}$ ) were isolated from plasma sequentially by a nonlinear salt gradient procedure described by Lindgren (13).

Polyacrylamide gel electrophoresis of the VLDL subfractions from two hypercholesterolemic and two control subjects was performed with analytical IEF of gels prepared in $8 \mathrm{M}$ urea with pH 4-6.5 ampholines (LKB, Sweden) as described by Cabana et al. (14) to identify isoforms of apolipoproteins E, CII, and CIII. Student's $t$ test was used to determine the significance of the differences of the mean values observed in CET and lipoprotein composition in the control and hypercholesterolemic groups.

\section{Results}

Cholesteryl ester transfer. The CET responses of the hypercholesterolemic (HC) patients and controls differed markedly (Fig. 1) during the 6-h incubation of whole plasma. In contrast to the initial delay and slow curvilinear increase in CET observed in controls, the $\mathrm{HC}$ group demonstrated a rapid initial increment and an overall hyperbolic response to levels that were significantly greater than those of controls at $1,2(P<0.001)$, and $4 \mathrm{~h}$ $(P<0.005)$. To assure that the differences observed in CET between $\mathrm{HC}$ and control subjects were not attributable to variations in the extent to which LCAT was inhibited, LCAT activity was assayed during the CET assay employing labeled free cholesterol and no radioactivity was recovered in $\mathrm{CE}$ in either HC or control groups.

Further studies were carried out to determine whether this alteration in CET resulted from changes in the acceptor VLDL and LDL, donor HDL, or CETP. First, experiments were performed in which the $d<1.063$ fractions containing the acceptor lipoproteins from HC subjects were added to the $d>1.063$ fraction of their corresponding controls containing HDL and CETP, and the control $d<1.063$ fractions were combined with

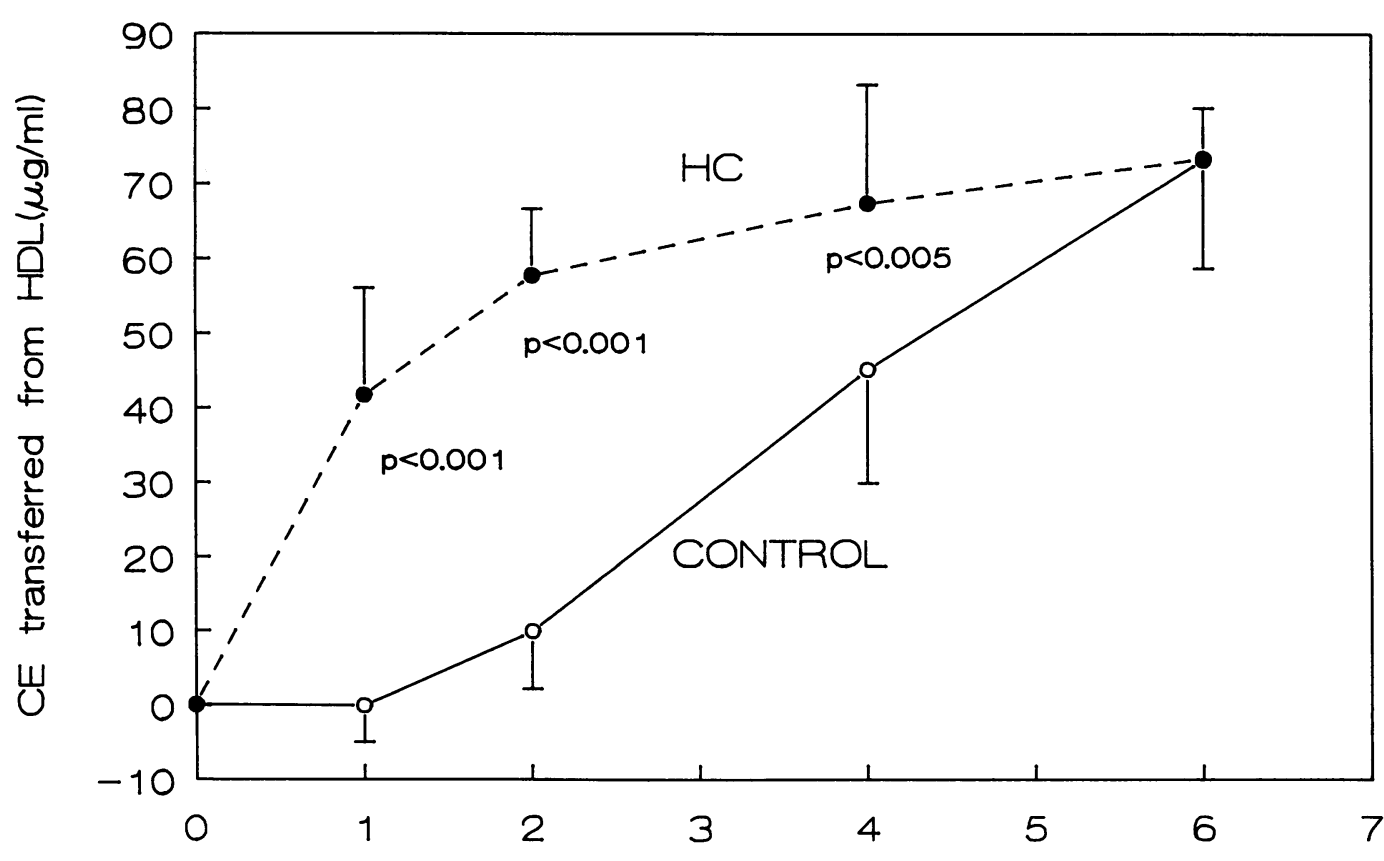

Figure 1. Mass of cholesteryl ester transferred from HDL to the apo Bcontaining lipoproteins in hypercholesterolemic $(H C)$ and control subjects. 


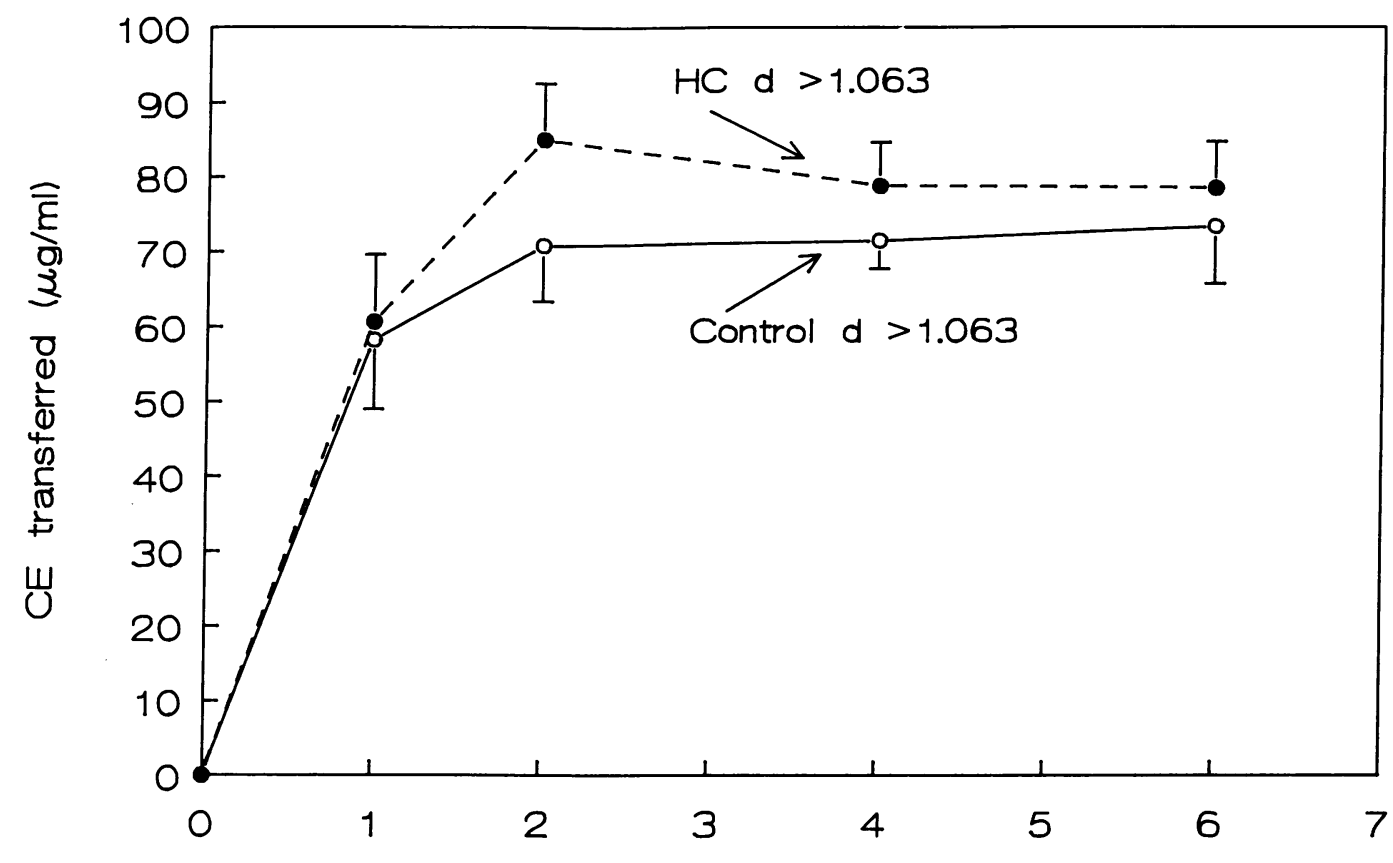

Hours
Figure 2. Mass of cholesteryl ester transferred when $d<1.063$ plasma fractions from six hypercholesterolemic subjects were combined with their own (open circles-solid line) and control (closed circles-dashed line) $d$ $>1.063$ plasma fractions. their respective $d>1.063 \mathrm{HC}$ fractions. Accelerated transfer identical to that present in intact HC plasma was observed with the combination containing $\mathrm{HC} d<1.063$ fraction (Fig. 2); in contrast, combining $\mathrm{HC} d>1.063$ plasma fractions with their respective control's $d<1.063$ fractions yielded CET responses that were indistinguishable from the intact control system (Fig. 3). The mass of CETP in the plasma of $11 \mathrm{HC}$ patients was significantly higher than controls $(\mathrm{HC}, 2.3 \pm \mu \mathrm{g} / \mathrm{ml}$ vs. control, $1.3 \pm .3 \mu \mathrm{g} / \mathrm{ml}$; mean $\pm \mathrm{SD} ; P<0.025)$. The fact that the $\mathrm{HC} d$
$>1.063$ fractions containing this increased concentration of CETP failed to accelerate CET when combined with control $d$ $<1.063$ lipoproteins, suggested that the acceleration observed in the $\mathrm{HC}$ group was related to alterations in their acceptor lipoproteins rather than to the increase in CETP.

To ascertain which of the acceptor lipoproteins was responsible for accelerating CET, VLDL and LDL from six HC patients were isolated and then added to the $d>1.063$ fractions of their respective controls. Combining their LDL with control $d$

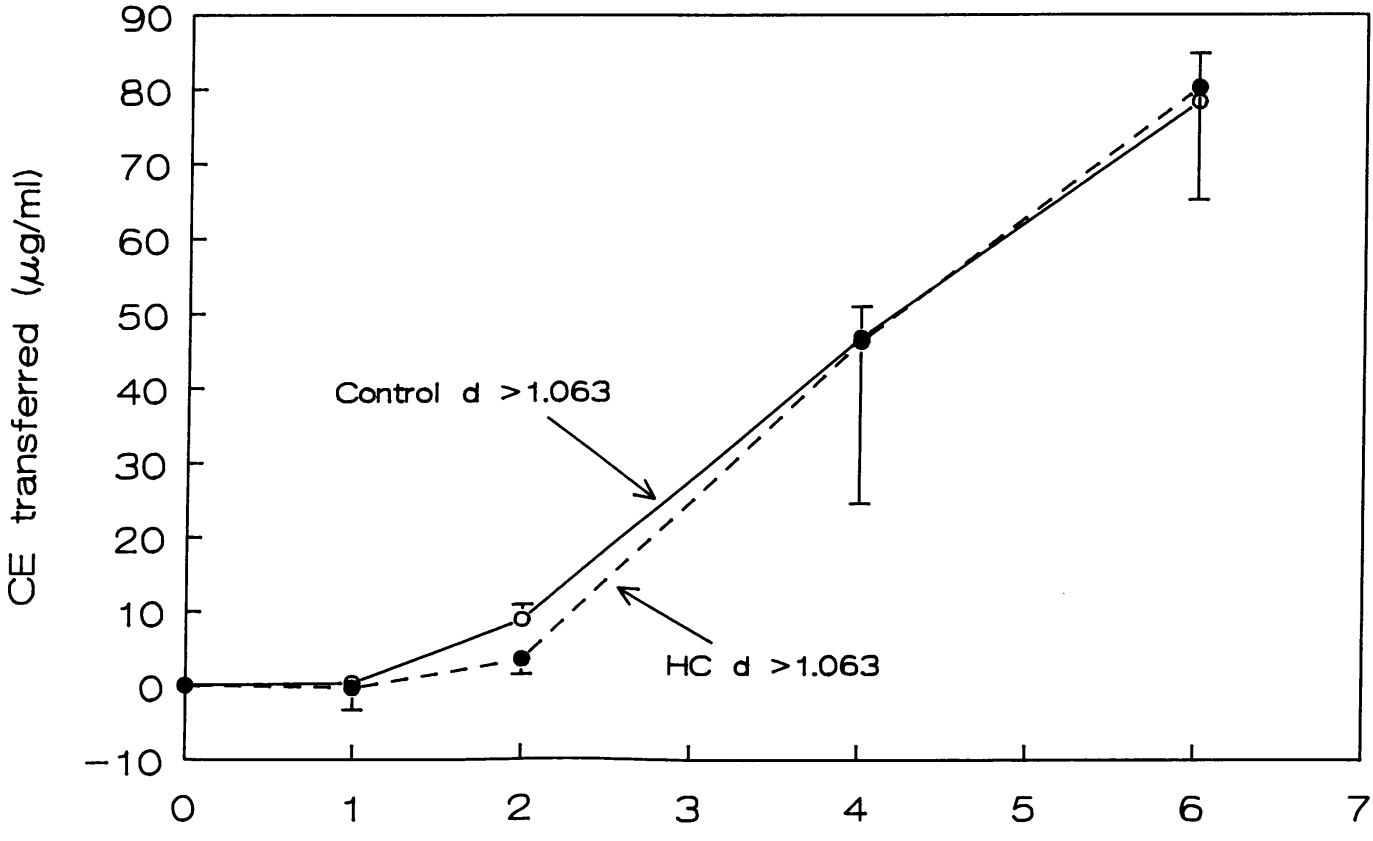

Hours
Figure 3. Mass of cholesteryl ester transferred when $d>1.063$ plasma fractions from six hypercholesterolemic patients were combined with $d$ $<1.063$ plasma fractions from controls (closed circles-dashed line) and reconstituted control system shown (open circlessolid line). 
Table I. Effects on Cholesteryl Ester Transfer* of Recombination of LDL from Six Hypercholesterolemic Patients and Control Subjects with $d>1.063$ Bottom Fractions from Controls

\begin{tabular}{lcccc}
\hline & \multicolumn{5}{c}{ Time } \\
\cline { 2 - 5 } & $1 \mathrm{~h}$ & $2 \mathrm{~h}$ & $4 \mathrm{~h}$ & $6 \mathrm{~h}$ \\
\hline $\begin{array}{c}\text { Control LDL + control } \\
\text { bottom }\end{array}$ & $5.5 \pm 10.2$ & $4.9 \pm 5.5$ & $0.8 \pm 9.4$ & $0.4 \pm 7.6$ \\
$\begin{array}{c}\text { Hypercholesterolemic LDL } \\
+ \text { control bottom }\end{array}$ & $-0.6 \pm 9.8$ & $0.6 \pm 10.2$ & $1.5 \pm 6.3$ & $4.0 \pm 3.6$
\end{tabular}

${ }^{*} \mu \mathrm{g} / \mathrm{ml} \mathrm{CE}$ transferred from $\mathrm{HDL} / \mathrm{ml}$ plasma. Values are mean $\pm \mathrm{SD}$.

$>1.063$ yielded CET activities that were identical to those observed when controls' $d 1.063$ top and bottom fractions were reconstituted (Table I). In contrast, HC VLDL plus controls' $d$ $>1.063$ fractions reproduced the pattern of accelerated CET observed in intact HC plasma (Fig. 4). This combination of observations indicated that this functional disturbance in CET in the HC patients was related to alterations in their VLDL and not in their LDL. To discern whether this stimulatory effect was common to one or more of the VLDL subfractions, $\mathrm{VLDL}_{1}, \mathrm{VLDL}_{2}$, and $\mathrm{VLDL}_{3}$ were isolated and each was added to simultaneously prepared control $d>1.063$ fractions. In these studies, accelerated transfer activity was consistently associated with VLDL $_{1}$ and not the other two VLDL subfractions (Fig. 5). This stimulatory effect was observed both when $\mathrm{VLDL}_{1}$ from two $\mathrm{HC}$ patients was added to the control $d$ $>1.063$ fraction at their respective plasma concentrations and at the same TG concentration as control $\mathrm{VLDL}_{1}$ (data not shown).

Lipoprotein composition. To determine whether alterations in lipoprotein composition were present which might contribute to this enhancement of CET in HC plasma, lipoprotein lipids from HC and control subjects were analyzed (Table II). The mass of all lipids tended to be higher in HC VLDL than in controls. The TG/CE ratio was lower $(P<0.1)$ and the free cholesterol $(\mathrm{FC}) /$ lecithin ratio significantly higher $(P<0.025)$ in the HC VLDL than in controls. (Table II). In HC LDL, all lipids were significantly increased reflecting the increase in LDL mass. The TG/CE and FC/lecithin ratios of HC LDL, however, both were significantly lower than those of the controls. HDL lipids were similar in the two groups, but the TG/ CE ratio tended to be higher in HC HDL than in controls. All three control VLDL subfractions (Table III) contained less lipid (total, free and esterified cholesterol, and triglyceride) than the corresponding $\mathrm{HC}$ subfractions. The major differences in these constituents were in $\mathrm{VLDL}_{1}$. Here the TG/CE ratio was significantly reduced $(P<0.001)$ and the $\mathrm{FC} /$ lecithin ratio increased $(P<0.05)$, indicating that its core was enriched in $\mathrm{CE}$ and its surface in FC; these ratios were very similar in $\mathrm{HC}$ and control $\mathrm{VLDL}_{2}$ and $\mathrm{VLDL}_{3}$. Analysis by PAGE with isoelectric focusing revealed no quantitative differences in the isoforms of apoproteins E, CII, and CIII in the VLDL subfractions.

\section{Discussion}

During the metabolism of lipoproteins in plasma, three parallel systems normally function to modify both their core and surface lipid composition. These include: (a) LCAT, which generates CE on HDL from free cholesterol acquired from other lipoproteins, the blood elements, and peripheral tissues (15); (b) specialized transfer proteins, which not only facilitate the exchange of core $\mathrm{CE}$ and triglyceride, but also serve to redistribute the phospholipid constituents among the lipoproteins (2); and $(c)$ the lipoprotein lipases, which remove triglyceride from the core of VLDL and chylomicrons, and remodel $\mathrm{HDL}_{2}$ through their lipolytic actions on both surface phospholipid and core triglyceride (16). HDL occupies a central place in each

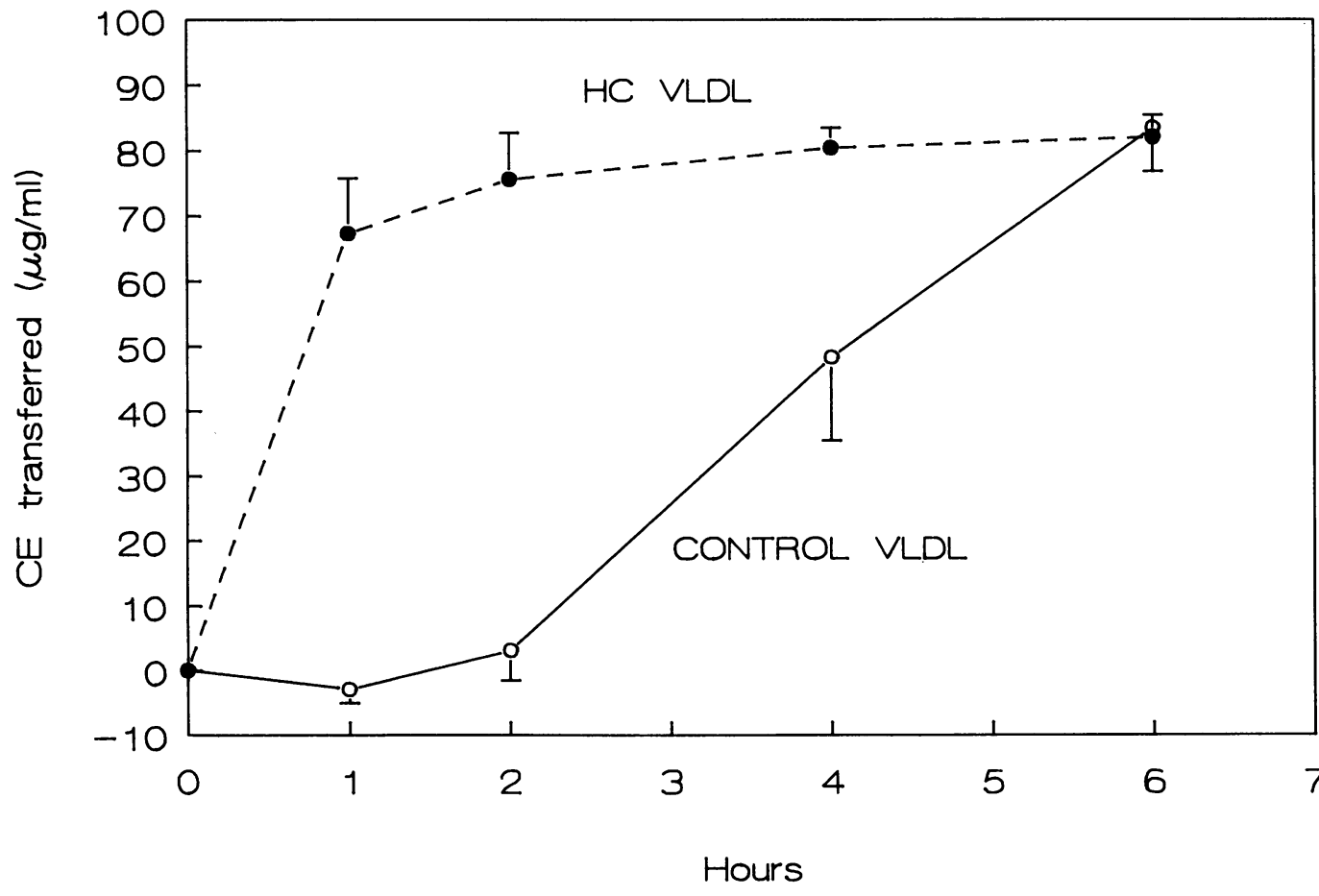

Figure 4. Mass of cholesteryl ester transferred from HDL when hypercholesterolemic (solid circles-dashed line) and control VLDL (open circlessolid line) were combined with $d>1.063$ plasma fractions from controls. 

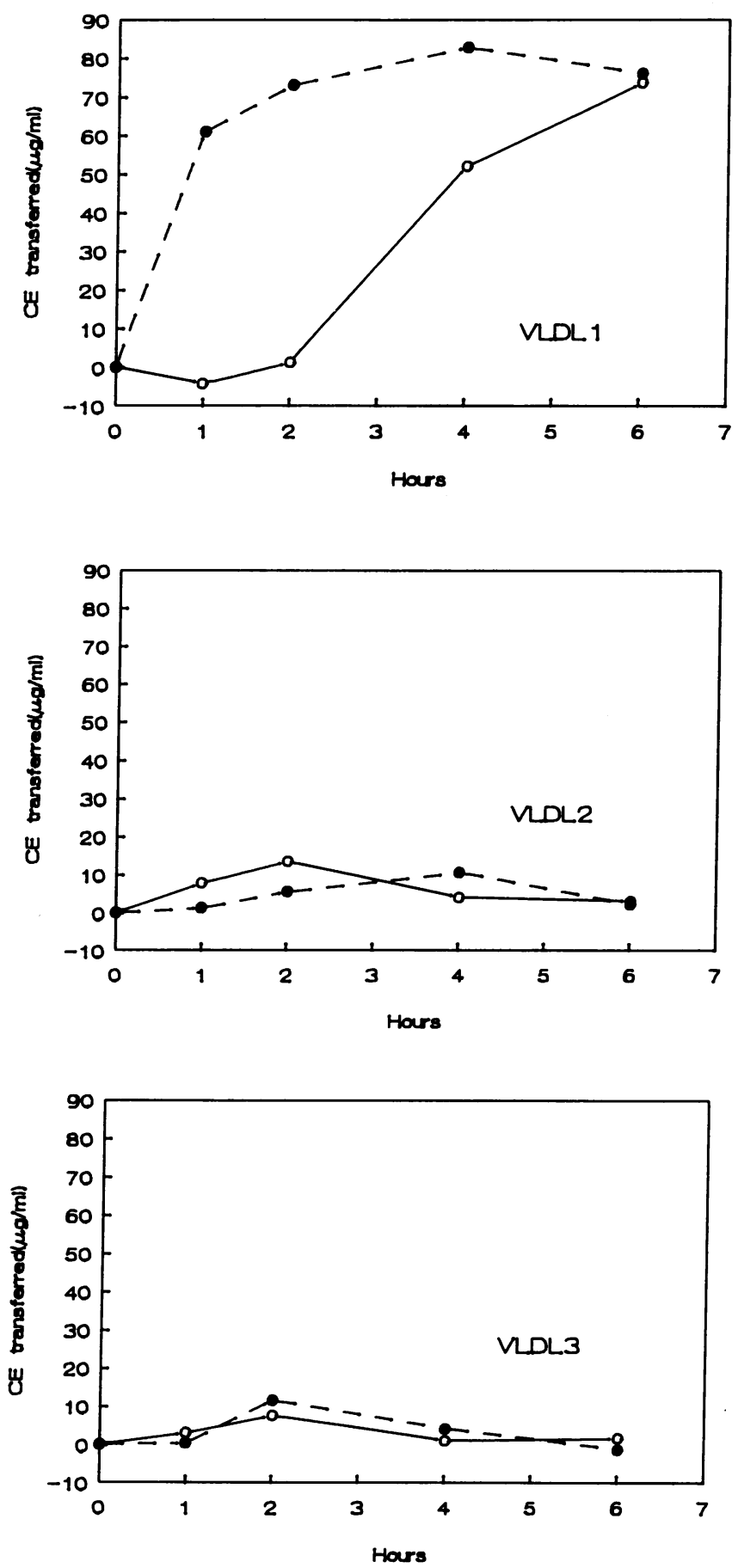

Figure 5. Mass of cholesteryl ester transferred from HDL when control $d>1.063$ plasma fractions were combined with $\mathrm{VLDL}_{1,2,3}$ from three hypercholesterolemic patients (solid circles-dashed line) and controls (open circles-solid line).

of these regulatory systems because it is the primary acceptor of FC, the principal site of its esterification, the donor of $C E$ to other lipoproteins, the acceptor of TG from VLDL, and a substate for hepatic lipase. How effectively HDL functions in these roles is believed to be the basis for it correlating inversely with the development of coronary heart disease (17).

In this study, we found that the initial rate of CE transferred from HDL to the apo B-containing lipoproteins in whole plasma is accelerated rather than inhibited as has been previously reported in hypercholesterolemic subjects (18). While this is a new observation in HC patients with the assay method employed, enhanced CET has been reported recently in similar HC patients $(19,20)$ and in cholesterol-fed rabbits (21) using isotopic techniques. In these earlier studies, however, the increase in CET was ascribed to increased activity of CETP, rather than to dysfunction of the acceptor or donor lipoproteins. In another recent report in which a similar increase in CET has been described in patients with dyslipidemia employing the same assay of mass transfer used in this study (22), the abnormality was also attributed to increased CETP activity. The series of recombination experiments we have performed to discern the mechanism underlying this disturbance in this cohort of HC patients, showed that it was the acceptor lipoprotein VLDL and not the increase we observed in CETP mass or in LDL or HDL, which was responsible for their acceleration of CET.

These observations suggest that the composition of VLDL rather than CETP concentration is a major determinant of the rate of CET. This possibility is supported by the observation that in the postprandial state, when the CET activity of plasma normally increases two- to threefold (9), there is only a small (10\%) increase in CET mass (12). Since our assay estimated only the mass of CE transferred from HDL that exchanged for TG, it is not surprising that VLDL and not LDL was associated with the increase in transfer activity (23), and that $\mathrm{VLDL}_{1}$, the most TG-enriched of the VLDL subfractions, was dysfunctional. Our data do not rule out the possibility, however, that homoexchange of CE between HDL and LDL is also accelerated.

The reasons for our finding that VLDL rather than CETP drives CET abnormally, as described in these other reports, may relate in part to marked differences in assay conditions; foremost among these is the fact that VLDL function in CET was not examined. The isotopic method employed by Groener et al. to study hyperlipidemic patients earlier (19) for example, involved the 16-h incubation of delipidated HC plasma with a standard mixture of radiolabeled HDL CE and LDL from a control pool; in these studies LDL rather than VLDL, which is normally the predominant $\mathrm{CE}$ acceptor, was used as an acceptor lipoprotein particle. In this study, we have measured the mass of CE transferred from each subjects' own HDL to their VLDL + LDL either in intact plasma or in a system containing freshly isolated lipoproteins from the same $\mathrm{HC}$ and control subjects.

The dissociation we observed between the mass of HC $\mathrm{VLDL}_{1}$ and its CET activity is noteworthy. While its cholesterol content was two- to threefold higher than control VLDL and its TG content increased by about one-half, its capacity to stimulate CET was more than 50-fold greater than that of control $\mathrm{VLDL}_{1}$. This stimulatory effect was demonstrable both when $\mathrm{HC} \mathrm{VLDL}_{1}$ was added to the controls' $d>1.063$ fraction at plasma concentration and at the same TG concentration as control $\mathrm{VLDL}_{1}$. Our compositional studies are consistent with accelerated CET taking place in $\mathrm{HC}$ patients in vivo. Specifically, the increase in the TG/CE ratio in HDL, which approached but did not quite reach statistical significance, and the clear cut reciprocal reduction found in $\mathrm{VLDL}_{1}$ in the $\mathrm{HC}$ patients are precisely the type of changes in core lipids to be expected if CET were increased.

One possible technical explanation for the differences we 
Table II. Lipoprotein Surface and Core Lipid Composition in Control and Hypercholesterolemic Subjects ${ }^{\mathrm{c}}$

\begin{tabular}{|c|c|c|c|c|c|c|c|}
\hline & TC & FC & CE & TG & $\frac{\mathrm{TG}}{\mathrm{CE}}$ & $\frac{\text { FC }}{\text { Lecithin }}$ & Lecithin \\
\hline & \multicolumn{4}{|c|}{$m g / d l$} & $m g / m g$ & $\mathrm{~mol} / \mathrm{mol}$ & $\mu \mathrm{mol} / \mathrm{ml}$ \\
\hline \multicolumn{8}{|l|}{ VLDL } \\
\hline HC & $14.5 \pm 11.7$ & $7.3 \pm 6.1$ & $7.2 \pm 5.9$ & $63.8 \pm 34.3$ & $9.3 \pm 2.8$ & $1.15 \pm 0.05^{\ddagger}$ & $0.20 \pm 0.13$ \\
\hline Control & $8.0 \pm 4.4$ & $4.4 \pm 2.0$ & $4.1 \pm 2.7$ & $47.1 \pm 24.9$ & $12.1 \pm 4.1$ & $0.78 \pm 0.14$ & $0.16 \pm 0.08$ \\
\hline \multicolumn{8}{|l|}{ LDL } \\
\hline HC & $224.0 \pm 61.7^{\prime \prime}$ & $52.1 \pm 5.6^{11}$ & $171.0 \pm 59.4^{\prime \prime}$ & $49.2 \pm 17.0^{8}$ & $0.32 \pm 0.17^{*}$ & $0.19 \pm 0.31^{8}$ & $1.18 \pm 0.28^{\prime \prime}$ \\
\hline Control & $83.0 \pm 12.9$ & $30.1 \pm 7.3$ & $53.1 \pm 9.9$ & $28.0 \pm 17.7$ & $0.53 \pm 0.32$ & $1.82 \pm 0.71$ & $0.48 \pm 0.16$ \\
\hline \multicolumn{8}{|l|}{ HDL } \\
\hline $\mathrm{HC}$ & $47.5 \pm 9.8$ & $11.8 \pm 2.4$ & $35.6 \pm 8.5$ & $16.3 \pm 5.4$ & $0.49 \pm 0.23$ & $0.39 \pm 0.07$ & $0.81 \pm 0.23$ \\
\hline Control & $45.9 \pm 8.0$ & $13.4 \pm 3.5$ & $32.5 \pm 6.5$ & $12.8 \pm 5.5$ & $0.37 \pm 0.15$ & $0.46 \pm 0.13$ & $0.74 \pm 0.14$ \\
\hline
\end{tabular}

TC, total cholesterol. ${ }^{*} P<0.05 ;{ }^{\ddagger} P<0.025 ;^{8} P<0.01$; and " $P<0.001$. ${ }^{\mathrm{c}}$ Controls, $n=12$; hypercholesterolemic subjects, $n=14$. Values are mean \pm SD.

find in CET, is that the sulfhydryl inhibitor DTNB employed to inhibit LCAT during the CET assay may have only partially inhibited cholesterol esterification in control plasma, but completely blocked it in the HC patients. If this were the case, the accumulation of newly synthesized CE in HDL would obscure the transfer of CE that had taken place, which we estimate as the mass of CE lost from HDL. This however, does not appear to be the case, since direct measurements of LCAT activity performed serially during the assay indicate that a comparable degree of inhibition was achieved in $\mathrm{HC}$ and control plasma with the concentration of DTNB employed. In preliminary experiments in two $\mathrm{HC}$ and two control subjects, we found similar differences in CET in whole plasma employing the serine protease inhibitor E-600 to inhibit LCAT (data not shown).

Another factor possibly contributing to increased CET in the $\mathrm{HC}$ subjects is an enrichment of their acceptor lipoproteins (VLDL + LDL) with lipolytic products or free fatty acids (FFA), as Tall et al. have shown, occurs normally in the postprandial state when CET is activated (9). FFA in HC VLDL could be increased de novo as a result of in vivo changes, or in vitro during our assay due to the phospholipase activity of LCAT which might not be fully inhibited by DTNB (24). Our finding that neither FFA nor lysolecithin increased makes it unlikely that the phospholipase activity of LCAT contributed significantly to the accelerated activity we observed. Since it has not been possible in our hands to reliably measure the very small amount of FFA present in $\mathrm{VLDL}_{1}$ with either GLC or colorimetric methods, a small increment in the FFA content of

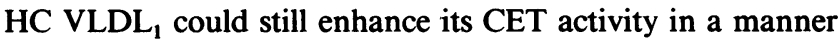
similar to that shown by Sammett and Tall for the entire VLDL fraction after treatment with milk lipase (25). This increased transfer activity of $\mathrm{VLDL}_{1}$ might also result from alterations in composition that enhance its avidity for CETP (26). Further studies are required to assess this possibility.

The FC content of VLDL relative to phospholipid has been shown to be a potent modifier of CET activity (4). It is therefore of interest that we found a directional change in the FC/lecithin ratio in $\mathrm{HC} \mathrm{VLDL}_{1}$ similar to that shown earlier by Morton to increase CET $(4,26)$. This finding may provide another possible explanation for the acceleration of CET we observe in HC patient. This abnormality may contribute to the formation of a subpopulation of VLDL which are abnormally enriched in CE. Particles generated in this way may be potentially atherogenic because they are preferentially cleared by arterial wall macrophages (27). These findings suggest that abnormalities in lipid transfer may accelerate atherogenesis in $\mathrm{HC}$ patients by adversely affecting the composition of their quantitatively normal VLDL.

Table III. VLDL Subfraction Lipid Composition in 12 Hypercholesterolemic and Control Subjects

\begin{tabular}{|c|c|c|c|c|c|c|c|}
\hline VLDL & TC & FC & CE & TG & $\frac{\mathrm{TG}}{\mathrm{CE}}$ & $\frac{\text { FC }}{\text { Lecithin }}$ & Lecithin \\
\hline & & & & & $m g / m g$ & $\mathrm{~mol} / \mathrm{mol}$ & $\mu \mathrm{mol} / \mathrm{ml}$ \\
\hline \multicolumn{8}{|l|}{ VLDL $_{1}$} \\
\hline $\mathrm{HC}$ & $3.9 \pm 2.5^{8}$ & $1.42 \pm 1.0^{\ddagger}$ & $2.5 \pm 1.5^{\ddagger}$ & $34.9 \pm 21.4^{\ddagger}$ & $14.1 \pm 4.2^{8}$ & $1.01 \pm 0.6^{*}$ & $0.053 \pm 0.04^{\ddagger}$ \\
\hline Control & $1.4 \pm 1.0$ & $0.61 \pm 0.4$ & $0.77 \pm 0.6$ & $17.2 \pm 12.5$ & $23.5 \pm 4.5$ & $0.72 \pm 0.5$ & $0.025 \pm 0.02$ \\
\hline \multicolumn{8}{|l|}{$\mathrm{VLDL}_{2}$} \\
\hline $\mathrm{HC}$ & $4.4 \pm 3.0$ & $1.8 \pm 1.4$ & $2.6 \pm 1.7^{*}$ & $27.1 \pm 14.9$ & $12.2 \pm 4.2$ & $0.84 \pm 0.4$ & $0.061 \pm 0.04$ \\
\hline Control & $2.6 \pm 1.5$ & $1.1 \pm 0.8$ & $1.4 \pm 0.8$ & $17.2 \pm 9.7$ & $12.4 \pm 3.4$ & $0.95 \pm 0.7$ & $0.040 \pm 0.03$ \\
\hline \multicolumn{8}{|l|}{$\mathrm{VLDL}_{3}$} \\
\hline $\mathrm{HC}$ & $9.1 \pm 4.2$ & $3.1 \pm 1.6$ & $6.0 \pm 3.1$ & $24.2 \pm 9.6$ & $4.4 \pm 1.6$ & $0.96 \pm 0.3$ & $0.102 \pm 0.07$ \\
\hline Control & $5.9 \pm 4.5$ & $2.1 \pm 1.6$ & $3.8 \pm 3.0$ & $17.1 \pm 11.0$ & $4.8 \pm 1.9$ & $1.01 \pm 0.9$ & $0.057 \pm 0.04$ \\
\hline
\end{tabular}

TC, total cholesterol. ${ }^{*} P<0.05 ;{ }^{\ddagger} P<0.025 ;$ and ${ }^{\S} P<0.001$. 


\section{Acknowledgments}

The authors are grateful to Dr. Alan Tall and Dr. Yves Marcel for performing the measurements of cholesteryl ester transfer protein. Thanks are also extended to Dr. Veneracion Cabana, Dr. Evan Stein, and to Dr. Michael Davidson and his associates at the Chicago Center for Clinical Research for their logistical support, to Wilfred Buchanan for technical assistance, and to Beverly Stoltz and Andrea P. Morrissey for manuscript preparation. This study was supported by grants from the National Institutes of Health HL-36073 and HL-39194.

\section{References}

1. Nichols, A. V., and L. Smith. 1965. Effect of very low density lipoproteins on lipid transfer in incubated serum. J. Clin. Invest. 6:206-210.

2. Tall, A. R. 1986. Plasma lipid transfer proteins. J. Lipid Res. 27:361-367.

3. Subbaiah, P. V., M. H. Davidson, M. C. Ritter, W. Buchanan, and J. D. Bagdade. 1989. Effects of dietary supplementation with marine lipid concentrate on the plasma lipoprotein composition of hypercholesterolemic patients. Atherosclerosis. 79:157-166.

4. Morton, R. E. 1988. Free cholesterol is a potent regulator of lipid transfer protein function. J. Biol. Chem. 263:12235-12241.

5. Rifkind, B. M., and P. Segal. 1983. Lipid Research Clinics Program reference values for hyperlipidemia and hypolipidemia. JAMA (J. Am. Med. Assoc.). 250:1869-1872.

6. Lipid Research Clinics Program. 1974. Manual of Laboratory Operations. Vol. I. Lipid and Lipoprotein Analysis. United States Government Printing Office, Washington, DC. D.H.E.W. Publication No. (NIH) 75-628.

7. Bligh, E. G., and W. J. Dyer. 1959. A rapid method of total lipid extraction and purification. Can. J. Biochem. Physiol. 37:911-917.

8. Marinetti, G. V. 1962. Chromatographic separation, identification, and analysis of phosphatides. J. Lipid Res. 3:1-20.

9. Tall, A. R., D. Sammett, and E. Granot. 1986. Mechanisms of enhanced cholesteryl ester transfer from high density lipoproteins to apo B-containing lipoproteins during alimentary lipemia. J. Clin. Invest. 77:1 163-1172.

10. Warnick, G. R., T. Nguyen, and J. J. Albers. 1985. Comparison of im proved precipitation methods for cholesterol quantitation of high-density lipoprotein. Clin. Chem. 31:217-222.

11. Gibson, J. C., A. Rubenstein, and W. V. Brown. 1984. Precipitation of apo E-containing lipoproteins by precipitation reagents for apolipoprotein B. Clin. Chem. 30:1784-1788.
12. Marcel Y. L., R. McPherson, M. Hogue, H. Czarnecka, Z. Zawadski, P. K Weech, M. E. Whitlock, A. R. Tall, and R. W. Milne. 1990 Distribution and concentration of cholesteryl ester transfer protein in plasma of normolipidemic subjects. J. Clin. Invest. 85:10-17.

13. Lindgren, F. T., L. C. Jensen, and F. T. Hatch. 1972. Blood Lipids and Lipoproteins: Quantitation, Composition, and Metabolism. G. J. Nelson, editor. Wiley-Interscience, New York. 181-274.

14. Cabana, J. G., J. N. Siegel, and S. Sabesin. 1989. Effects of the acute phase response on the concentration and density distribution of plasma lipids and apoliproteins. J. Lipid Res. 130:39-49.

15. Glomset, J. A., and K. R. Norum. 1973. The metabolic role of lecithin cholesterol acyltransferase. Perspectives from Pathology. Adv. Lipid Res. 1:1-65.

16. Deckelbaum, R. D., E. Granot, Y. Oschry, L. Rose, and S. Eisenberg 1984. Plasma triglyceride determines structure composition in low and high density lipoproteins. Arteriosclerosis. 4:225-231.

17. Eisenberg, S. 1985. High density lipoprotein metabolism. J. Lipid Res. 25:1017-1058.

18. Fielding, P. E., C. J. Fielding, R. J. Havel, J. P. Kane, and Tun P. 1983. Cholesterol net transport, esterification, and transfer in human hyperlipidemic plasma. J. Clin. Invest. 71:449-460.

19. Groener, J. E. M., A. J. Van Rozen, and D. W. Erkelens. 1984. Cholesteryl ester transfer activity, localization and role in distribution of cholesteryl ester among lipoproteins in man. Atherosclerosis. 50:261-271.

20. Inazu, A., J. Koizumi, Y. Uno, H. Fujita, K. Kajinami, M. Takeda, and H. Ito. 1988. Enhanced cholesteryl ester transfer activity in heterozygous familial hypercholesterolemia. Proceedings of the 8th International Symposium on Atherosclerosis. Rome, October 9-13. p. 400.

21. Son, Y.C., and D. B. Zilversmit. 1986. Increased lipid transfer activities in hyperlipidemic rabbit plasma. Atherosclerosis. 6:345-355.

22. Tall, A., E. Granot, R. Brocia, I. Tabas, C. Hesler, K. Williams, and M. Denke. 1987. Accelerated transfer of cholesteryl esters in dyslipidemic plasma. Role of cholesterol ester transfer protein. J. Clin. Invest. 79:1217-1225.

23. Eisenberg, S. 1985. Preferential enrichment of large-sized very low density lipoprotein populations with transferred cholesteryl esters. J. Lipid Res. 26:487494.

24. Dolphin, P. J., and H. Jauhianen. 1987. Human plasma lecithin-cholesterol acyltransferase-the catalytic site and mechanism of action. Atheroma (Lisbon). 13:16-28.

25. Sammet, D., and A. R. Tall. 1985. Mechanisms of enhancement of cholesteryl ester transfer protein activity by lipolysis. J. Biol. Chem. 260:6687-6697.

26. Morton, R. E. 1990 . Interaction of lipid transfer protein with plasma lipoproteins and cell membranes. Experientia. 46:552-561.

27. Mahley, R. W. 1982. Atherogenic hyperlipoproteinemia. The cellular and molecular biology of plasma lipoproteins altered by dietary fat and cholesterol. Med. Clin. N. Am. 66:375-401. 